\title{
INEQUALITIES FOR ENTIRE FUNCTIONS OF EXPONENTIAL TYPE
}

\author{
T. GENCHEV
}

\begin{abstract}
DEDICATED TO THE MEMORY OF N. OBRESHKov (1896-1963)-MEMBER OF
\end{abstract} THE Bulgarian ACADEMY OF SCIENCES

\begin{abstract}
This paper is concerned with a class of linear operators acting in the space of the trigonometric polynomials and preserving the inequalities of the form $|S(\theta)|<|T(\theta)|$ in the half plane $\operatorname{Im} \theta>0$. Some inequalities for entire functions of exponential type and some theorems concerning the distribution of the zeros of the trigonometric polynomials, including an analogue to the Gauss-Lucas theorem, are derived.
\end{abstract}

1. Introduction. Using interpolation series, R. P. Boas [1] obtains the following interesting extension of the classic S. Bernstein inequality:

THEOREM. Let $f(z)$ be an entire function of exponential type $\sigma$ with $|f(z)| \leqq M$ on the real axis. Then the inequality

$$
\left|f(x+i y) e^{-i w}+f(x-i y) e^{i w}\right| \leqq 2 M\left(\cosh ^{2} \sigma y-\sin ^{2} w\right)^{1 / 2}, \quad w \quad \text { real },
$$

holds.

This theorem, as shown by Boas himself [1], has a number of important consequences. Our purpose is to give a new proof and some extension of (1). At the same time our method, which is based on a principle suggested by a paper of De Bruijn [2], and on a theorem of Obreshkov [3] concerning the zeros of the rational polynomials, allows us to prove some theorems about the zeros of the trigonometric polynomials, including a theorem analogous to the classic Gauss-Lucas theorem.

2. The principle mentioned above is given by

THEOREM 1. Let $\Re$ be a closed subset of the complex plane $C$ and let $\mathfrak{N}$ be a complex linear space of meromorphic functions with poles in $\%$ Further, let $L: \Re \rightarrow \pi$ be a linear operator and $\Re$ the subset of $\Re$ consisting of the functions having no zeros in $C \backslash \mathcal{K}_{0}$. Then the inequality $|f(z)|<|g(z)|, z \in C \backslash \mathcal{K}, f, g$ $\in \mathfrak{T}$, implies the inequality $|L(f)(z)|<|L(g)(z)|, z \in C \backslash \mathcal{K}$, if and only if $L(\Re) \subset \Re$

Proof. Let us suppose that $L(\Re) \subset \Re$ and $|f(z)|<|g(z)|$ in $C \backslash \mathcal{K}$, but nevertheless, there exists $z_{0} \in C \backslash \mathcal{K}$ such that $\left|L(f)\left(z_{0}\right)\right| \geqq\left|L(g)\left(z_{0}\right)\right|$. Intro-

Received by the editors October 4, 1974.

AMS (MOS) subject classifications (1970). Primary 30A04, 30A08; Secondary 30A40.

Key words and phrases. Inequalities in the complex domain, trigonometric polynomials, extremal problems. 
ducing the functions $f-\lambda g$, where $\lambda=L(f)\left(z_{0}\right)\left[L(g)\left(z_{0}\right)\right]^{-1}$ ( $\lambda$ is well defined because $g \in \Re$ implies $L(g) \neq 0$ in $C \backslash \mathcal{K}$ ), we obviously have $f-\lambda g \in \Re$, since $|\lambda| \geqq 1$. Thus $L(f-\lambda g)=L(f)-\lambda L(g) \in \Re$. This is impossible, however, because $L(f-\lambda g)\left(z_{0}\right)=0$.

Considering the pair of $f, g$, where $f \equiv 0$ and $g \in \mathcal{R}$ is arbitrary, we conclude that the condition $L(\Re) \subset \Re$ is necessary.

In the sequel, except Theorem 1, we shall need a slight modification of the following elementary result due to Obreshkov [3].

THEOREM 2. Let $\Phi$ be the strip bounded by two parallel lines making angles of $\phi$ with the real axis and let all the zeros of the rational polynomial $f(z)$ lie in D. Then all the zeros of the polynomial

$$
F(z)=f(z+h)-\gamma f(z-h) \quad \text { where }|\gamma|=1 \text { and } \arg h=\phi+\pi / 2,
$$

also lie in $\mathscr{D}$.

Because of the importance of this theorem for our considerations, we shall outline the proof of Obreshkov.

Proof. Let $z_{1}, z_{2}, \ldots, z_{n}$ be the zeros of $f(z)$ and let $z_{0}$ be a zero of $F(z)$. Then we have

$$
\left|\frac{f\left(z_{0}+h\right)}{f\left(z_{0}-h\right)}\right|=1 \text { i.e. } \prod_{k=1}^{n}\left|\frac{z_{0}+h-z_{k}}{z_{0}-h-z_{k}}\right|=1 .
$$

Supposing for a moment that $z_{0}$ lies outside $\mathscr{D}$, we immediately come to a contradiction, because all the factors $\left|\left(z_{0}+h-z_{k}\right) /\left(z_{0}-h-z_{k}\right)\right|, k=1,2$, $\ldots, n$, are simultaneously less than or greater than 1 .

The same reasoning proves

THEOREM 3. Let all the zeros of the rational polynomial $f(z)$ lie in the half plane $\operatorname{Im} z \leqq a$ and let $h$ and $k, 0 \leqq k \leqq h, h>0$, be real numbers. Then the zeros of the polynomial $f(z+h i)-\gamma f(z-k i),|\gamma| \leqq 1$, also lie in $\operatorname{Im} z \leqq a$.

It will be convenient for our purpose to introduce the following

Definition. A trigonometric polynomial of the form

$$
T(\theta)=\sum_{\nu=-n}^{n} a_{\nu} e^{i \nu \theta}, \quad a_{-n} \neq 0,
$$

having no zeros in the half plane $H: \operatorname{Im} \theta>0$ is said to belong to class $P_{n}$.

(In this paper by a trigonometric polynomial of degree not exceeding $n$ we always mean an expression of the form (2) without any restriction on the coefficients.)

REMARK. By means of the substitution $w=e^{i \theta}$ and the maximum principle, it is easily seen that in $H$ the inequality $|\bar{T}(\theta)| \leqq|T(\theta)|$ is satisfied, where $\bar{T}(\theta)=\sum_{\nu=-n}^{n} \bar{a}_{\nu} e^{-i \nu \theta}$ and $\bar{a}_{\nu}$ is the conjugate of $a_{\nu}$. Consequently we have $T(-\theta) \in P$, where $P$ is the class of the majorants studied by $B$. Levin and others [4, p. 129].

Now we are in a position to prove our main theorem.

THEOREM 4. Let $S(\theta)$ and $T(\theta)$ be trigonometric polynomials of degree not exceeding $n$ and $T(\theta) \in P_{n}$. Furthermore, let $S(\theta)$ and $T(\theta)$ be linearly independ- 
ent and satisfy the inequality $|S(\theta)| \leqq|T(\theta)|$ on the real axis. Then the inequality

$$
|S(\theta+\lambda i)-\tau S(\theta-\mu i)|<|T(\theta+\lambda i)-\tau T(\theta-\mu i)|, \quad \operatorname{Im} \theta>0,
$$

where $0 \leqq \mu \leqq \lambda, \lambda>0$ and $|\tau| \leqq(\cosh (\lambda / 2) / \cosh (\mu / 2))^{2 n}$, is satisfied.

Proof. First of all by means of the substitution $w=e^{i \theta}$ and the maximum principle, we derive the inequality $|S(\theta)|<|T(\theta)|$ for $\theta \in H$. Furthermore, introducing polynomials $S_{1}(\theta)=S(\theta+\alpha), T_{1}(\theta)=T(\theta+\alpha)$, where $\alpha \in H$ is arbitrary and fixed, we obviously have

$$
\left|S_{1}(\theta)\right|<\left|T_{1}(\theta)\right| \text { for } \operatorname{Im} \theta>-\operatorname{Im} \alpha .
$$

Now, setting $z=\operatorname{tg}(\theta / 2)$, we obtain

$$
S_{1}(\theta)=P(z) /\left(1+z^{2}\right)^{n}, \quad T_{1}(\theta)=Q(z) /\left(1+z^{2}\right)^{n},
$$

where $P(z)$ and $Q(z)$ are rational polynomials of degree not exceeding $2 n$. Since the function $z=\operatorname{tg}(\theta / 2)$ maps $H$ to $H \backslash\{i\}$ and $Q(i)=4^{n} a_{-n} e^{-i n \alpha} \neq 0$, the inequality

$$
|P(z)|<|Q(z)|, \quad \operatorname{Im} z \geqq 0,
$$

follows from (4). Moreover, the relation

$$
\lim _{x \rightarrow \pm \infty}\left|\frac{P(x)}{Q(x)}\right|=\lim _{\theta \rightarrow \pm \pi}\left|\frac{S_{1}(\theta)}{T_{1}(\theta)}\right|=\left|\frac{S(\alpha \pm \pi)}{T(\alpha \pm \pi)}\right|<1, \quad x \text { real, }
$$

implies (6) in the half plane $\operatorname{Im} z \geqq-\varepsilon$, where $\varepsilon>0$ is sufficiently small.

In order to apply Theorem 1 let us denote by $\mathcal{K}$ the half plane $\operatorname{Im} z \leqq-\varepsilon$, where $\varepsilon>0$ is chosen so that (6) holds in $C \backslash \mathcal{K}_{0}$ Let $\pi$ be the complex space of rational polynomials of degree not exceeding $2 n$ and let $\Re$ be the subset of T consisting of the polynomials having no zeros outside $\xi_{0}$ According to Theorem 3, for the operator

$$
L(f)=f(z+h i)-\gamma f(z-k i), \quad 0 \leqq k \leqq h, h>0,|\gamma| \leqq 1, f \in \mathfrak{R},
$$

we have $L(\Re) \subset \Re$. Recalling (6) and applying Theorem 1 we obtain

$$
|P(z+h i)-\gamma P(z-k i)|<|Q(z+h i)-\gamma Q(z-k i)|
$$

in $C \backslash \Re$ and, in particular, in $\operatorname{Im} z \geqq 0$.

Now let the real numbers $\lambda, \mu, 0 \leqq \mu \leqq \lambda, \lambda>0$, be arbitrary. Setting $z=0, h=\operatorname{tgh}(\lambda / 2), k=\operatorname{tgh}(\mu / 2)$ in (7), by means of (5) we get

$$
\begin{aligned}
\mid S(\alpha+ & \lambda i)-\gamma\left(\frac{\cosh (\lambda / 2)}{\cosh (\mu / 2)}\right)^{2 n} S(\alpha-\mu i) \mid \\
& <\left|T(\alpha+\lambda i)-\gamma\left(\frac{\cosh (\lambda / 2)}{\cosh (\mu / 2)}\right)^{2 n} T(\alpha-\mu i)\right|,
\end{aligned}
$$

and since $\alpha \in H$ is arbitrary, the proof of Theorem 4 is complete.

REMARK. If we have $|S(\theta)|<|T(\theta)|$ on the real axis, then (3) is satisfied in 
the closed half plane $\operatorname{Im} \theta \geqq 0$. Indeed, it suffices to note that the inequality $|S(\theta)|<|T(\theta)|$ holds on the line $\operatorname{Im} \theta=-\varepsilon$, where $\varepsilon>0$ is sufficiently small, and to apply Theorem 4 to the pair $S(\theta-i \varepsilon), T(\theta-i \varepsilon)$.

COROLlary 1. Let us set $\tau=1$ in (3) and, after dividing by $\lambda-\mu$, let $\lambda \rightarrow 0$, $\mu \rightarrow 0$. Then we get the known inequality [4]

$$
\left|S^{\prime}(\theta)\right| \leqq\left|T^{\prime}(\theta)\right|, \quad \operatorname{Im} \theta \geqq 0,
$$

which obviously includes Bernstein's inequality. Furthermore, applying Theorem 4 with $\tau=e^{2 i w}, w$ real, to the pair $e^{-i w} S(\theta)$ and $e^{-i w} T(\theta)$, we obtain the inequality

$$
\begin{array}{r}
\left|e^{-i w} S(\theta+\lambda i)+e^{i w} S(\theta-\mu i)\right|<\left|e^{-i w} T(\theta+\lambda i)+e^{i w} T(\theta-\mu i)\right|, \\
\operatorname{Im} \theta>0,0 \leqq \mu \leqq \lambda,
\end{array}
$$

so that the linear operator $T(\theta) \rightarrow e^{-i w} T(\theta+\lambda i)+e^{i w} T(\theta-\mu i)$, acting on the space of the trigonometric polynomials, leaves the class $P_{n}$ invariant and preserves inequalities of the form $|S(\theta)|<|T(\theta)|$ in the half plane $\operatorname{Im} \theta>0$. Hence it is a $B$-operator in the sense of Levin $[4, p .226]$.

COROLlaRY 2. Let us consider the operator

$$
L_{f}(R)(\theta)=\sum_{\nu=0}^{m} C_{\nu} R(\theta+(m-\nu) \lambda i-\nu \mu i), \quad 0 \leqq \mu \leqq \lambda, \lambda>0,
$$

where $f(z)=\sum_{v=0}^{m} C_{v} z^{m-v}$ is fixed, $R(\theta)$ being a trigonometric polynomial. If all the zeros of $f(z)$ lie in the circle $|z| \leqq(\cosh (\lambda / 2) / \cosh (\mu / 2))^{2 n}$, the inequality

$$
\left|L_{f}(S)(\theta)\right|<\left|L_{f}(T)(\theta)\right|, \quad \operatorname{Im} \theta>0,
$$

is satisfied.

One may prove this corollary by applying Theorem 4 successively with $\tau=\gamma_{k}, k=1,2, \ldots, m$, where $\left\{\gamma_{k}\right\}$ are the zeros of $f(z)$.

In turn, inequality (10) implies

Corollary 3. If $T(\theta) \in P_{n}$, then $L_{f}(T)(\theta) \in P_{n}$.

Proof. Applying (10) to the pair $S(\theta) \equiv 0, T(\theta)$, we see that $L_{f}(T)(\theta)$ has no zeros in the half plane $\operatorname{Im} \theta>0$. Since $L_{f}(T)(\theta)$ obviously has the form (2), the corollary is proved.

Now we may state a theorem analogous to a theorem of L. Weisner [6].

THEOREM 5. If $T(\theta) \in P_{n}$, then

$$
L(T)(\theta)=\int_{\theta-\mu i}^{\theta+\lambda i} T(t) d t, \quad 0 \leqq \mu \leqq \lambda, \lambda>0,
$$

also belongs to $P_{n}$.

Proof. First of all it is immediately seen that $L(T)(\theta)$ has the form (2). Since the zeros of the polynomial $\sum_{\nu=0}^{m} z^{\nu}$ lie on the circle $|z|=1$, according to Corollary 3 the zeros of the Riemann sums 


$$
T_{m}(\theta)=\frac{(\lambda+\mu) i}{m} \sum_{\nu=1}^{m} T\left(\theta+\frac{\lambda i}{m}(m-\nu)-\frac{\mu \nu}{m} i\right)
$$

lie in $\operatorname{Im} \theta \leqq 0$ and the conclusion follows from the Hurwitz theorem.

THEOREM 6. If the conditions of Theorem 4 are satisfied, the inequality $|L(S)(\theta)|<|L(T)(\theta)|, \operatorname{Im} \theta>0$, where the operator $L$ is given by (11), holds.

PRoof. Let $\Re$ be the complex linear space of trigonometric polynomials of degree not exceeding $n$, and $\varkappa=P_{n}$. According to Theorem 5 we have $L(\Re) \subset \Re$ and we complete the proof by applying Theorem 1 .

Now we need the following

Definition. A trigonometric polynomial of the form

$$
T(\theta)=\sum_{\nu=-n}^{n} a_{\nu} e^{i \nu \theta}, \quad a_{n} a_{-n} \neq 0
$$

will be called balanced.

The following theorem is analogous to Theorem 2.

THEOREM 7. Let $T(\theta)$ be a balanced trigonometric polynomial with zeros in the strip $a \leqq \operatorname{Im} \theta \leqq b$. Then all the zeros of $N(\theta)=T(\theta+\lambda i)-\gamma T(\theta-\lambda i)$, $|\gamma|=1, \lambda>0$, also lie in this strip.

Proof. Obviously $T(\theta+b i)$ belongs to $P_{n}$. Applying Theorem 4 to the pair $S(\theta) \equiv 0$ and $T(\theta+b i)$, we conclude that $N(\theta) \neq 0$ in $\operatorname{Im} \theta>b$. Since $T(-\theta+a i) \in P_{n}$, by the same reasoning $N(\theta)$ has no zeros in $\operatorname{Im} \theta<a$.

COROLlaRY 1. (Gauss-Lucas theorem for trigonometric polynomials.) If $T(\theta)$ is balanced and has zeros only in the strip $\Omega: a \leqq \operatorname{Im} \theta \leqq b$, the zeros of its derivative $T^{\prime}(\theta)$ also lie in $\Omega$.

Proof. Letting $\lambda \rightarrow 0$ in $(T(\theta+\lambda i)-T(\theta-\lambda i)) / \lambda$ and applying Hurwitz's theorem, we obtain the proof.

Going into details, one could prove that $T^{\prime}(\theta)$ may have a zero $\theta_{0}$ on one of the lines $\operatorname{Im} \theta=a, \operatorname{Im} \theta=b$, such that $T\left(\theta_{0}\right) \neq 0$, if and only if all the zeros of $T(\theta)$ lie on the same line [5].

COROLlaRY 2. If $T(\theta)$ is balanced and has zeros only in the strip $\Omega$, then all the zeros of the trigonometric polynomial

$$
L(T)(\theta)=\int_{\theta-\lambda i}^{\theta+\lambda i} T(t) d t, \quad \lambda>0,
$$

also lie in $\Omega$.

Proof. This corollary can be deduced from Theorem 7 exactly as Theorem 5 was deduced from Corollary 3 of Theorem 4.

3. It is obvious that Theorem 4 , with $|\tau| \leqq 1$, could be extended to the case when $S(\theta)$ and $T(\theta)$ are entire functions of exponential type belonging to appropriate classes. Here, for the sake of brevity, we shall confine ourselves to deriving some consequences of Theorem 4 concerning entire functions of exponential type bounded on the real axis, including Boas' inequality. 
Let $f(z)$ be an entire function of exponential type $\sigma$, subject to the condition $|f(z)| \leqq M$ on the real axis. According to a theorem of B. Levitan [7, p. 193], there exists a sequence of trigonometric polynomials $S_{n}(z)$ $=\sum_{\nu=-n}^{n} a_{\nu, n} e^{-i \nu \sigma z / n}$, tending uniformly to $f(z)$ on every bounded set, and such that the inequality $\left|S_{n}(z)\right| \leqq M$ is satisfied on the real axis. Applying (9) to the pair $S_{n}((n / \sigma) \theta)$ and $M e^{-i n \theta}$, we obtain

$$
\begin{gathered}
\left|e^{-i w} S_{n}\left(\frac{n}{\sigma}\left(\theta_{n}+\lambda_{n} i\right)\right)+e^{i w} S_{n}\left(\frac{n}{\sigma}\left(\theta_{n}-\mu_{n} i\right)\right)\right| \\
\leqq M\left|e^{-i n \theta_{n}}\right|\left|e^{-i w+n \lambda_{n}}+e^{i w-n \mu_{n}}\right|
\end{gathered}
$$

where $\theta_{n}=\sigma z / n, \operatorname{Im} z \geqq 0, \lambda_{n}=\sigma \lambda / n, \mu_{n}=\sigma \mu / n, 0 \leqq \mu \leqq \lambda, \lambda>0$.

Letting $n \rightarrow \infty$ in (12) we get

$$
\begin{aligned}
& \left|e^{-i w} f(z+\lambda i)+e^{i w} f(z-\mu i)\right| \\
& \quad \leqq M\left|e^{-i w} e^{-i \sigma(z+\lambda i)}+e^{i w} e^{-i \sigma(z-\mu i)}\right|, \quad 0 \leqq \mu \leqq \lambda, \operatorname{Im} z \geqq 0,
\end{aligned}
$$

in which Boas' inequality is included.

Finally, applying (13) twice with $w=\pi / 2, z$ real, $\lambda=|y|, \mu=0$ to the functions $f(z)$ and $f(-z)$, we obtain

$$
|f(x+i y)-f(x)| \leqq M\left(e^{o|y|}-1\right), \quad x, y \text { real, }
$$

from which Bernstein's inequality follows again.

In the same way we deduce from Theorem 6 the inequality

$$
\left|\int_{z-\mu i}^{z+\lambda i} f(t) d t\right| \leqq M\left|\int_{z-\mu i}^{z+\lambda i} e^{-i \sigma t} d t\right| \leqq \frac{M}{\sigma}\left|e^{\sigma(y+\lambda)}-e^{\sigma(y-\mu)}\right|
$$

where $0 \leqq \mu \leqq \lambda, y=\operatorname{Im} z \geqq 0$, and of course, $|f(z)| \leqq M$ on the real axis.

The inequalities (14) and (15) are obviously exact.

\section{REFERENCES}

1. R. P. Boas, Inequalities for functions of exponential type, Math. Scand. 4 (1956), 29-32. MR 19, 24.

2. N. G. de Bruijn, Inequalities concerning polynomials in the complex domain, Nederl. Acad. Wetensch. Proc. 50 (1947), 1265-1272 = Indag. Math. 9 (1947), 591-598. MR 9, 347.

3. N. Obrechkoff, Sur les racines des equations algébriques, Tôhoku Math. J. 38 (1933), 93100.

4. R. P. Boas, Jr., Entire functions, Academic Press, New York, 1954. MR 16, 914.

5. T. Genchev, A Gauss-Lucas type theorem on trigonometric polynomials, C.R. Acad. Sci. Bulgare 28 (1975), 449-451.

6. L. Weisner, On the regional location of the zeros of certain functions, Tôhoku Math. J. 44 (1937), 175-177.

7. N. I. Ahiezer, Lectures on the theory of approximation, 2nd rev. ed., "Nauka", Moscow, 1965; English transl. of 1st ed., Ungar, New York, 1956. MR 20 \#1872; 32 \#108.

Mathematics Faculty of Sofia University, Boul. A. Ivanov 5, Sofia 1126, Bulgaria 